\title{
Weed Management Studies in Wheat (Triticum aestivum) with Herbicides under Different Planting Methods
}

\author{
Navish Kumar Kamboj ${ }^{1 *}$, V.S. Hooda ${ }^{1}$, Gaurendra Gupta $^{2}$ and Meenakshi Sangwan ${ }^{1}$ \\ ${ }^{1}$ Department of Agronomy, CCSHAU, Hisar-125004, HR, India \\ ${ }^{2}$ Division of Agronomy, IARI, New Delhi \\ *Corresponding author
}

\section{A B S T R A C T}

\section{Keywords}

Wheat, Planting methods, Bed planting, Zero tillage, Herbicide, Weed management.

Article Info

Accepted: 24 January 2017 Available Online: 10 February 2017
A field experiment was conducted during rabi season of 2013-14 to study the performance of wheat under different planting methods with varied row spacing and herbicides. Experiment was laid out in split-plot design having six planting methods [bed planting with two and three rows, drill sowing at 18 and $20 \mathrm{~cm}$ (conventional tillage), drill sowing at 18 and $20 \mathrm{~cm}$ (zero tillage)] as main-plot treatments and five herbicidal treatments [pinoxaden $50 \mathrm{~g} / \mathrm{ha}$, \{carfentrazone + metsulfuron $(\mathrm{RM})$ \} $25 \mathrm{~g} / \mathrm{ha}$, pinoxaden + \{carfentrazone + metsulfuron $(\mathrm{RM})\}(50+25 \mathrm{~g}) / \mathrm{ha}$, weed free and weedy check] as subplot treatments replicated thrice. Various planting methods resulted in significant difference in population and dry matter accumulation by weeds from 60 DAS to harvest. Maximum wheat yield and minimum numbers of weeds and their lowest dry matter accumulation were observed in zero tillage, whereas, lower wheat yield and maximum number of weeds and their highest dry matter accumulation were observed in bed planting method. Density of weeds and their dry matter accumulation increased with increasing row spacing from 18 $\mathrm{cm}$ (drill sowing both in ZT and CT) to $35 \mathrm{~cm}$ (bed planting with two rows), whereas, the crop yield increased with decreased row spacing. Among the various herbicidal treatments, the combined application of pinoxaden + [carfentrazone + metsulfuron $(\mathrm{RM})](50+25 \mathrm{~g})$ /ha was most effective against both grassy and broadleaf weeds with more than 90 per cent weed control efficiency (WCE \%). Pinoxaden $50 \mathrm{~g} / \mathrm{ha}$, significantly reduced the population and dry matter accumulation of grassy weeds having 93 per cent WCE for grassy weeds, whereas, application of carfentrazone + metsulfuron (RM) (25 g/ha), significantly reduced the population and dry matter accumulation of all broad leaved weeds with more than 95 per cent WCE.

\section{Introduction}

Wheat, in India, is second to rice in terms of area and production, producing 90-95 $\mathrm{mt}$ of wheat from 29-31 $\mathrm{m}$ ha area. Rice-wheat is the most common and important crop sequence in the Indo-Gangetic plains with more than $13.5 \mathrm{~m}$ ha area. In Haryana wheat was produced in about $2.5 \mathrm{~m}$ ha area producing
$11.6 \mathrm{mt}$ with a productivity of $40.66 \mathrm{q} / \mathrm{ha}$ during 2014-15. Even though, we have almost reached a plateau in wheat productivity per unit area due to various biotic and abiotic factors, yet wheat is considered most assured crop in the country which is essential for country's nutritional security. Losses caused 
by weeds in wheat depend on the infesting weed type, its intensity, and agronomic practices adopted in wheat cultivation (Singh, 2007). Grassy weeds viz. littleseed canarygrass and wild oat have been the most prevalent weeds of wheat (Singh et al., 1995). Wild oat has been most competitive among the two, significantly reducing crop growth and productivity (Balyan et al., 1991).

Losses caused by weeds in wheat vary from 20-50 per cent, but there could be complete crop failure in extreme cases (Malik and Singh, 1995). Herbicide resistance is the major cause of yield loss as continuous use of herbicides of same site of action resulted in multiple herbicide resistance (Singh et al., 2009). The situation is further complicated due to shift in weed flora towards difficult to control weeds as a result of using the same agronomic practices (herbicides). Wheat is infested with both grassy and broadleaf weeds and effective weed management require an integrated approach using both chemical and non-chemical approaches (Chhokar et al., 2012 and Singh, 2007). For effective management of complex weed flora, mixture of more than one herbicide is required. Herbicide mixtures not only increase weed control efficacy against complex weed flora (Singh et al., 2011), they are also useful in delaying herbicide resistance (Wrubel and Gressel, 1994).

The mixture should have a wide spectrum of weed control without crop injury and no residual effect on succeeding sensitive crops after wheat. Crop-weed interference is affected by cultural practices like method of sowing, crop density and geometry. Some of the agronomic techniques such as, variable plant density (Kapler et al., 2002) and crop geometrics (Sharma and Angiras, 1996) can be effectively integrated with herbicide to allow the crop-weed competition in favour of crops and reduce load of herbicides per unit area which will ultimately helps in sustaining agriculture. Therefore, there is a need to focus on integrated weed management and succession of weeds in a cropping sequence. Keeping the above mentioned points in view, the present experiment was conducted to evaluate the effects different planting methods and herbicides on weeds and yield of wheat crop.

\section{Materials and Methods}

The experiment was conducted during rabi season of 2013-14 at Research Area of Agronomy, CCS Haryana Agricultural University, Hisar, Haryana (India) situated at $29^{\circ} 10^{\prime} \mathrm{N}$ latitude and $75^{\circ} 46^{\prime} \mathrm{E}$ longitude at an elevation of $215.2 \mathrm{~m}$ above mean sea level to study the performance of wheat under different herbicides and planting methods with varied row spacing.

The soil of the experimental field was sandy loam in texture, low in organic carbon and nitrogen, medium in available phosphorus, high in potassium and slightly alkaline $(\mathrm{pH}$ 8.4) in reaction. Experiment was laid out in split-plot design replicated thrice, having six planting methods as main-plot treatments, viz. bed planting with two and three rows, drill sowing at 18 and $20 \mathrm{~cm}$ (conventional tillage), drill sowing at 18 and $20 \mathrm{~cm}$ (zero tillage) and five herbicidal treatments as subplot treatments, viz. pinoxaden $50 \mathrm{~g} / \mathrm{ha}$, [carfentrazone + metsulfuron (RM)] $25 \mathrm{~g} / \mathrm{ha}$, pinoxaden + [carfentrazone + metsulfuron (RM)] $(50+25 \mathrm{~g}) / \mathrm{ha}$, weed free and weedy check. Wheat variety HD-2967 was sown on $3^{\text {rd }}$ December, 2013 and the crop was raised as per package of practices recommended by CCS Haryana Agricultural University, Hisar, except herbicidal treatment.

Herbicides as per treatments were sprayed at 35 DAS using 500 1/ha water by knapsack sprayer fitted with flat-fan nozzle. Weed population and their dry weight was recorded periodically (Table 1, $2 \& 3$ ) using $1 \mathrm{~m}^{3}$ 
quadrate. The dry weight of weeds was recorded (sun dried) for the counted weeds from each plot and then kept them in oven at $70{ }^{\circ} \mathrm{C}$ till constant weight was achieved. Based on dry wt. of weeds, weed control efficiency (WCE) was calculated by the following formula:

WCE $(\%)=$

Dry wt. of weeds in untreated plot - Dry wt. of weeds in treated plot

Dry wt. of weeds in untreated plot

The experimental data for various weeds, their density along with their dry matter accumulation, WCE and crop yield were statistically analyzed by using the F -test (Gomez and Gomez, 1983). The significance of treatment effects were computed with the help of critical difference (CD) at $\mathrm{P}=0.05$ by determining the difference between mean values of treatments (Cochran and Cox, 1959).

\section{Results and Discussion}

Effect of planting methods on weeds and crop

The experimental field was infested with natural population of Phalaris minor, Chenopodium album, Anagallis arvensis and Melilotus indica and other miscellaneous weeds (Avena ludoviciana, Coronopus didymus, Lathyrus aphaca, Cirsium arvense, Medicago denticulata, Medicago hispida, Fumaria parviflora, Vicia sp. and Cynodon dactylon).

Weed count was done at 30 DAS to observe infestation and distribution of weeds flora in the experimental field before application of herbicides. The results (Tables 1 and 2) revealed that at 30 DAS, various planting methods had no significant difference in respect of weed count. This was mainly due to the fact that at initial stage, crop canopy development was almost similar under all planting methods as enough space was available between the rows. From 60 DAS to crop harvest, the density and dry matter accumulation of weeds (both grassy and broad leaved weeds) increased with increasing row spacing from $18 \mathrm{~cm}$ (drill sowing both in ZT and CT) to $35 \mathrm{~cm}$ (bed planting with two rows).

Minimum numbers of weeds and their dry matter accumulation were observed in zero tillage at $18 \mathrm{~cm}$ row spacing and maximum in bed planting with two rows (Table 1, 2 and 3). The higher density and dry matter accumulation of weeds in bed planting with two rows of wheat was on account of more space available in furrows as well as on beds, while in bed planting with three rows of wheat only vacant space was available between the beds i.e. in furrows. The density of weeds was more under bed planted wheat (FIRBS) followed by drill sowing in CT and lowest in drill sowing in ZT.

Dev et al., (2013) also reported less weed density and their dry matter accumulation under zero tillage as compared to conventional tillage. From 60 DAS to crop harvest, the density and dry matter accumulation of weeds in bed planting with two rows remained significantly higher than all other planting methods whereas density and dry matter accumulation of weeds in bed planting with three rows remained at par with $20 \mathrm{~cm}$ row spacing (both in ZT and CT) but significantly higher than $18 \mathrm{~cm}$ row spacing (both in ZT and CT). Less dry matter accumulation by weeds under closer row spacing of $15 \mathrm{~cm}$ in wheat was also reported by Walia et al., (2003) against wider row spacing (20 to $22.5 \mathrm{~cm}$ ) in comparison to other planting methods like bed planting with two and three rows and flat sowing with 22.5 $\mathrm{cm}$ row spacing. 
Table.1 Effect of different herbicides and planting methods in wheat on weed density (No./m2)

\begin{tabular}{|c|c|c|c|c|c|c|c|c|c|c|c|c|c|c|c|}
\hline \multirow[t]{2}{*}{ Treatments } & \multicolumn{5}{|c|}{ Phalaris minor $\left(\mathrm{No} . / \mathrm{m}^{2}\right)$} & \multicolumn{5}{|c|}{ Chenopodium album (No. $\left./ \mathrm{m}^{2}\right)$} & \multicolumn{5}{|c|}{ Anagallis arvensis $\left(\mathrm{No} . / \mathrm{m}^{2}\right)$} \\
\hline & $\begin{array}{c}\text { 30 } \\
\text { DAS }\end{array}$ & $\begin{array}{c}\text { 60 } \\
\text { DAS }\end{array}$ & $\begin{array}{c}90 \\
\text { DAS }\end{array}$ & $\begin{array}{l}120 \\
\text { DAS }\end{array}$ & $\begin{array}{c}\text { At } \\
\text { harvest }\end{array}$ & $\begin{array}{c}\text { 30 } \\
\text { DAS }\end{array}$ & $\begin{array}{c}\text { 60 } \\
\text { DAS } \\
\end{array}$ & $\begin{array}{c}90 \\
\text { DAS } \\
\end{array}$ & $\begin{array}{l}120 \\
\text { DAS }\end{array}$ & \begin{tabular}{|c|} 
At \\
harvest
\end{tabular} & $\begin{array}{c}30 \\
\text { DAS } \\
\end{array}$ & $\begin{array}{c}\text { 60 } \\
\text { DAS } \\
\end{array}$ & $\begin{array}{c}90 \\
\text { DAS }\end{array}$ & $\begin{array}{c}120 \\
\text { DAS } \\
\end{array}$ & $\begin{array}{c}\text { At } \\
\text { harvest }\end{array}$ \\
\hline \multicolumn{16}{|l|}{ Planting methods } \\
\hline $\begin{array}{l}\text { Drill sowing at } 20 \\
\mathrm{~cm}(\mathrm{CT})\end{array}$ & $\begin{array}{r}5.45 \\
(33.53) \\
\end{array}$ & $\begin{array}{c}3.60 \\
(18.20) \\
\end{array}$ & $\begin{array}{c}3.68 \\
(19.20) \\
\end{array}$ & $\begin{array}{c}3.48 \\
(16.93) \\
\end{array}$ & $\begin{array}{c}3.45 \\
(16.33) \\
\end{array}$ & $\begin{array}{c}7.76 \\
(70.87) \\
\end{array}$ & $\begin{array}{c}3.91 \\
(22.13) \\
\end{array}$ & $\begin{array}{c}3.78 \\
(20.21) \\
\end{array}$ & $\begin{array}{c}3.40 \\
(16.01) \\
\end{array}$ & $\begin{array}{c}3.28 \\
(14.53) \\
\end{array}$ & \begin{tabular}{|c|}
4.25 \\
$(20.60)$ \\
\end{tabular} & $\begin{array}{c}6.09 \\
(62.80) \\
\end{array}$ & $\begin{array}{c}4.98 \\
(40.20)\end{array}$ & \begin{tabular}{|c|}
4.59 \\
$(34.47)$ \\
\end{tabular} & $\begin{array}{c}4.30 \\
(30.00) \\
\end{array}$ \\
\hline $\begin{array}{l}\text { Drill sowing at } 18 \\
\mathrm{~cm}(\mathrm{CT})\end{array}$ & $\begin{array}{c}5.37 \\
(32.67)\end{array}$ & $\begin{array}{c}3.54 \\
(17.01)\end{array}$ & $\begin{array}{c}3.61 \\
(17.93)\end{array}$ & $\begin{array}{c}3.43 \\
(15.80)\end{array}$ & $\begin{array}{c}3.39 \\
(15.27)\end{array}$ & $\begin{array}{c}7.66 \\
(68.93)\end{array}$ & $\begin{array}{c}3.85 \\
(20.59)\end{array}$ & $\begin{array}{c}3.70 \\
(18.79) \\
\end{array}$ & $\begin{array}{c}3.33 \\
(14.81)\end{array}$ & $\begin{array}{c}3.21 \\
(13.40)\end{array}$ & $\begin{array}{c}4.16 \\
(19.73) \\
\end{array}$ & $\begin{array}{c}6.08 \\
(62.53)\end{array}$ & $\begin{array}{c}4.96 \\
(40.13) \\
\end{array}$ & \begin{tabular}{|c|}
4.58 \\
$(34.33)$ \\
\end{tabular} & $\begin{array}{c}4.27 \\
(29.87)\end{array}$ \\
\hline $\begin{array}{l}\text { Drill sowing at } 20 \\
\mathrm{~cm}(\mathrm{ZT})\end{array}$ & $\begin{array}{c}5.35 \\
(32.40)\end{array}$ & $\begin{array}{c}3.57 \\
(17.47)\end{array}$ & $\begin{array}{c}3.64 \\
(18.40)\end{array}$ & $\begin{array}{c}3.46 \\
(16.20)\end{array}$ & $\begin{array}{c}3.42 \\
(15.67)\end{array}$ & $\begin{array}{c}7.63 \\
(68.40)\end{array}$ & $\begin{array}{c}3.89 \\
(21.08)\end{array}$ & $\begin{array}{c}3.73 \\
(19.25)\end{array}$ & $\begin{array}{c}3.37 \\
(15.30)\end{array}$ & $\begin{array}{c}3.25 \\
(13.80)\end{array}$ & $\begin{array}{c}4.14 \\
(19.53)\end{array}$ & $\begin{array}{c}6.06 \\
(61.80)\end{array}$ & $\begin{array}{c}4.93 \\
(39.53)\end{array}$ & $\begin{array}{c}4.55 \\
(33.80)\end{array}$ & $\begin{array}{c}4.25 \\
(29.33)\end{array}$ \\
\hline $\begin{array}{l}\text { Drill sowing at } 18 \\
\mathrm{~cm}(\mathrm{ZT})\end{array}$ & $\begin{array}{c}5.43 \\
(33.53) \\
\end{array}$ & $\begin{array}{c}3.49 \\
(16.27) \\
\end{array}$ & $\begin{array}{c}3.56 \\
(17.20)\end{array}$ & $\begin{array}{c}3.39 \\
(15.33)\end{array}$ & $\begin{array}{c}3.35 \\
(14.67) \\
\end{array}$ & $\begin{array}{c}7.75 \\
(70.73)\end{array}$ & $\begin{array}{c}3.79 \\
(19.73) \\
\end{array}$ & $\begin{array}{c}3.68 \\
(17.95) \\
\end{array}$ & $\begin{array}{c}3.31 \\
(14.13) \\
\end{array}$ & $\begin{array}{c}3.20 \\
(12.87)\end{array}$ & \begin{tabular}{|c|}
4.15 \\
$(19.72)$
\end{tabular} & $\begin{array}{c}5.99 \\
(61.53) \\
\end{array}$ & $\begin{array}{c}4.90 \\
(39.07)\end{array}$ & $\begin{array}{c}4.51 \\
(33.60)\end{array}$ & $\begin{array}{c}4.20 \\
(29.13) \\
\end{array}$ \\
\hline $\begin{array}{l}\text { Bed planting (3 } \\
\text { rows) }\end{array}$ & $\begin{array}{c}5.40 \\
(33.07) \\
\end{array}$ & $\begin{array}{c}3.68 \\
(19.13) \\
\end{array}$ & $\begin{array}{c}3.76 \\
(20.27) \\
\end{array}$ & $\begin{array}{c}3.57 \\
(17.93) \\
\end{array}$ & $\begin{array}{c}3.52 \\
(17.27) \\
\end{array}$ & $\begin{array}{c}7.71 \\
(69.87) \\
\end{array}$ & $\begin{array}{c}4.02 \\
(23.16) \\
\end{array}$ & $\begin{array}{c}3.84 \\
(21.14) \\
\end{array}$ & $\begin{array}{c}3.47 \\
(16.81) \\
\end{array}$ & $\begin{array}{c}3.34 \\
(15.21) \\
\end{array}$ & \begin{tabular}{|c|}
4.20 \\
$(19.93)$ \\
\end{tabular} & $\begin{array}{c}6.14 \\
(62.87) \\
\end{array}$ & \begin{tabular}{|c|}
5.01 \\
$(40.22)$ \\
\end{tabular} & \begin{tabular}{|c|}
4.63 \\
$(34.53)$ \\
\end{tabular} & $\begin{array}{c}4.32 \\
(30.73)\end{array}$ \\
\hline $\begin{array}{l}\text { Bed planting (2 } \\
\text { rows) }\end{array}$ & $\begin{array}{c}5.43 \\
(33.47) \\
\end{array}$ & $\begin{array}{c}4.09 \\
(24.93) \\
\end{array}$ & $\begin{array}{c}4.18 \\
(26.40) \\
\end{array}$ & $\begin{array}{c}3.96 \\
(23.20) \\
\end{array}$ & $\begin{array}{c}3.91 \\
(22.33) \\
\end{array}$ & $\begin{array}{c}7.75 \\
(70.67) \\
\end{array}$ & $\begin{array}{c}4.43 \\
(30.03) \\
\end{array}$ & $\begin{array}{c}4.25 \\
(27.40) \\
\end{array}$ & $\begin{array}{c}3.83 \\
(21.67) \\
\end{array}$ & $\begin{array}{c}3.68 \\
(19.60) \\
\end{array}$ & $\begin{array}{c}4.26 \\
(20.87) \\
\end{array}$ & \begin{tabular}{|c|}
6.29 \\
$(65.80)$ \\
\end{tabular} & $\begin{array}{c}5.16 \\
(42.33) \\
\end{array}$ & \begin{tabular}{|c|}
4.74 \\
$(36.47)$ \\
\end{tabular} & $\begin{array}{c}4.40 \\
(31.67) \\
\end{array}$ \\
\hline SEm \pm & 0.04 & 0.03 & 0.03 & 0.03 & 0.03 & 0.06 & 0.04 & 0.04 & 0.03 & 0.03 & 0.04 & 0.03 & 0.02 & 0.02 & 0.03 \\
\hline $\mathrm{CD}$ at $5 \%$ & NS & 0.11 & 0.12 & 0.10 & 0.10 & NS & 0.13 & 0.11 & 0.10 & 0.09 & NS & 0.10 & 0.08 & 0.07 & 0.11 \\
\hline \multicolumn{16}{|c|}{ Weed control management } \\
\hline PDN (50 g/ha) & $\begin{array}{c}6.48 \\
(41.22) \\
\end{array}$ & $\begin{array}{c}2.06 \\
(3.33) \\
\end{array}$ & $\begin{array}{c}2.07 \\
(3.35) \\
\end{array}$ & $\begin{array}{c}1.99 \\
(3.00)\end{array}$ & $\begin{array}{c}1.97 \\
(2.98) \\
\end{array}$ & $\begin{array}{c}9.35 \\
(86.78)\end{array}$ & $\begin{array}{c}7.32 \\
(53.26) \\
\end{array}$ & $\begin{array}{c}7.00 \\
(48.62) \\
\end{array}$ & $\begin{array}{c}6.24 \\
(38.39) \\
\end{array}$ & $\begin{array}{c}5.92 \\
(34.5) \\
\end{array}$ & \begin{tabular}{|c|}
5.11 \\
$(26.67)$ \\
\end{tabular} & $\begin{array}{c}12.35 \\
(151.78) \\
\end{array}$ & $\begin{array}{c}9.93 \\
(97.67) \\
\end{array}$ & $\begin{array}{c}9.21 \\
(84.06) \\
\end{array}$ & $\begin{array}{c}8.60 \\
(73.50) \\
\end{array}$ \\
\hline $\begin{array}{l}\text { CZN+MSM } \\
(\mathrm{RM})(25 \mathrm{~g} / \mathrm{ha})\end{array}$ & $\begin{array}{c}6.42 \\
(40.33)\end{array}$ & $\begin{array}{c}6.67 \\
(44.06)\end{array}$ & $\begin{array}{c}6.86 \\
(46.67)\end{array}$ & $\begin{array}{c}6.44 \\
(40.94)\end{array}$ & $\begin{array}{c}6.32 \\
(39.39)\end{array}$ & $\begin{array}{c}9.28 \\
(85.33)\end{array}$ & $\begin{array}{c}2.22 \\
(4.08)\end{array}$ & $\begin{array}{c}2.14 \\
(3.72)\end{array}$ & $\begin{array}{c}1.94 \\
(2.89)\end{array}$ & $\begin{array}{c}1.92 \\
(2.87)\end{array}$ & $\begin{array}{c}4.75 \\
(23.28)\end{array}$ & $\begin{array}{c}2.32 \\
(4.56)\end{array}$ & \begin{tabular}{|c|}
3.03 \\
$(3.17)$
\end{tabular} & $\begin{array}{c}1.74 \\
(2.11)\end{array}$ & $\begin{array}{c}1.55 \\
(1.44)\end{array}$ \\
\hline $\begin{array}{l}\mathrm{PDN}+[\mathrm{CZN}+\mathrm{MSM} \\
(\mathrm{RM})] \\
(50+25 \mathrm{~g}) / \mathrm{ha}\end{array}$ & $\begin{array}{c}6.53 \\
(41.72)\end{array}$ & $\begin{array}{l}1.93 \\
(2.78)\end{array}$ & $\begin{array}{c}1.94 \\
(2.79)\end{array}$ & $\begin{array}{l}1.91 \\
(2.67)\end{array}$ & $\begin{array}{c}1.90 \\
(2.65)\end{array}$ & $\left(\begin{array}{c}9.43 \\
(88.06)\end{array}\right.$ & $\begin{array}{c}2.03 \\
(3.28)\end{array}$ & \begin{tabular}{|c|}
1.97 \\
$(2.99)$
\end{tabular} & $\begin{array}{c}1.87 \\
(2.56)\end{array}$ & $\begin{array}{c}1.85 \\
(2.55)\end{array}$ & $\begin{array}{c}4.78 \\
(23.56)\end{array}$ & $\begin{array}{c}2.50 \\
(5.33)\end{array}$ & $\begin{array}{c}2.18 \\
(3.83)\end{array}$ & $\begin{array}{c}1.85 \\
(2.50)\end{array}$ & $\begin{array}{l}1.65 \\
(1.78)\end{array}$ \\
\hline Weed free & $\begin{array}{c}1.00 \\
(0.00) \\
\end{array}$ & $\begin{array}{c}1.00 \\
(0.00)\end{array}$ & $\begin{array}{c}1.00 \\
(0.00) \\
\end{array}$ & $\begin{array}{c}1.00 \\
(0.00) \\
\end{array}$ & $\begin{array}{c}1.00 \\
(0.00) \\
\end{array}$ & $\begin{array}{c}1.00 \\
(0.00) \\
\end{array}$ & $\begin{array}{c}1.00 \\
(0.00) \\
\end{array}$ & $\begin{array}{c}1.00 \\
(0.00) \\
\end{array}$ & $\begin{array}{c}1.00 \\
(0.00) \\
\end{array}$ & $\begin{array}{c}1.00 \\
(0.00) \\
\end{array}$ & \begin{tabular}{|c|}
1.00 \\
$(0.00)$ \\
\end{tabular} & $\begin{array}{c}1.00 \\
(0.00) \\
\end{array}$ & $\begin{array}{c}1.00 \\
(0.00) \\
\end{array}$ & \begin{tabular}{|c|}
1.00 \\
$(0.00)$ \\
\end{tabular} & $\begin{array}{c}1.00 \\
(0.00) \\
\end{array}$ \\
\hline Weedy check & $\begin{array}{c}6.57 \\
(42.28) \\
\end{array}$ & $\begin{array}{c}6.65 \\
(44.00) \\
\end{array}$ & $\begin{array}{c}6.84 \\
(46.72) \\
\end{array}$ & $\begin{array}{c}6.43 \\
(41.06) \\
\end{array}$ & $\begin{array}{c}6.31 \\
(39.50) \\
\end{array}$ & $\begin{array}{c}9.50 \\
(89.40)\end{array}$ & $\begin{array}{c}7.30 \\
(53.27) \\
\end{array}$ & $\begin{array}{c}6.98 \\
(48.63) \\
\end{array}$ & $\begin{array}{c}6.21 \\
(38.33) \\
\end{array}$ & $\begin{array}{c}5.91 \\
(34.56) \\
\end{array}$ & $\begin{array}{c}5.13 \\
(26.83)\end{array}$ & $\begin{array}{c}12.37 \\
(152.78) \\
\end{array}$ & $\begin{array}{c}9.86 \\
(96.56)\end{array}$ & \begin{tabular}{|c|}
9.20 \\
$(83.83)$
\end{tabular} & $\begin{array}{c}8.64 \\
(73.89) \\
\end{array}$ \\
\hline SEm \pm & 0.07 & 0.14 & 0.14 & 0.13 & 0.12 & 0.10 & 0.15 & 0.15 & 0.13 & 0.12 & 0.16 & 0.15 & 0.11 & 0.09 & 0.13 \\
\hline $\mathrm{CD}$ at $5 \%$ & 0.19 & 0.39 & 0.40 & 0.36 & 0.35 & 0.28 & 0.44 & 0.42 & 0.37 & 0.35 & 0.47 & 0.43 & 0.30 & 0.27 & 0.37 \\
\hline
\end{tabular}


Table. 2 Effect of different herbicides and planting methods in wheat on weed density (No./m2)

\begin{tabular}{|c|c|c|c|c|c|c|c|c|c|c|}
\hline \multirow[t]{2}{*}{ Treatments } & \multicolumn{5}{|c|}{ Melilotus indica $\left(\mathrm{No} . / \mathrm{m}^{2}\right)$} & \multicolumn{5}{|c|}{ Miscellaneous weeds (No. $/ \mathbf{m}^{2}$ ) } \\
\hline & $\begin{array}{c}\text { 30 } \\
\text { DAS }\end{array}$ & $\begin{array}{c}\text { 60 } \\
\text { DAS }\end{array}$ & $\begin{array}{c}90 \\
\text { DAS }\end{array}$ & $\begin{array}{c}120 \\
\text { DAS }\end{array}$ & $\begin{array}{c}\text { At } \\
\text { harvest }\end{array}$ & $\begin{array}{c}\text { 30 } \\
\text { DAS }\end{array}$ & \begin{tabular}{|c|} 
60 \\
DAS
\end{tabular} & \begin{tabular}{|c|} 
90 \\
DAS \\
\end{tabular} & $\begin{array}{l}\text { 120 } \\
\text { DAS }\end{array}$ & $\begin{array}{c}\text { At } \\
\text { harvest }\end{array}$ \\
\hline \multicolumn{11}{|l|}{ Planting methods } \\
\hline $\begin{array}{l}\text { Drill sowing at } 20 \\
\mathrm{~cm}(\mathrm{CT})\end{array}$ & $\begin{array}{c}4.86 \\
(26.30)\end{array}$ & $\begin{array}{c}3.15 \\
(13.73)\end{array}$ & $\begin{array}{c}2.93 \\
(11.40)\end{array}$ & $\begin{array}{c}2.78 \\
(10.00)\end{array}$ & $\begin{array}{c}2.51 \\
(8.00)\end{array}$ & $\begin{array}{c}3.35 \\
(11.73)\end{array}$ & $\begin{array}{c}3.73 \\
(19.07)\end{array}$ & $\begin{array}{c}3.58 \\
(17.53)\end{array}$ & $\begin{array}{c}3.36 \\
(15.47)\end{array}$ & $\begin{array}{c}3.11 \\
(12.93)\end{array}$ \\
\hline $\begin{array}{l}\text { Drill sowing at } 18 \\
\mathrm{~cm}(\mathrm{CT})\end{array}$ & $\begin{array}{c}4.82 \\
(25.87) \\
\end{array}$ & $\begin{array}{c}3.08 \\
(13.15) \\
\end{array}$ & $\begin{array}{c}2.88 \\
(11.27) \\
\end{array}$ & $\begin{array}{c}2.74 \\
(9.80) \\
\end{array}$ & $\begin{array}{l}2.48 \\
(7.73) \\
\end{array}$ & $\begin{array}{c}3.32 \\
(11.47) \\
\end{array}$ & $\begin{array}{c}3.65 \\
(18.40) \\
\end{array}$ & $\begin{array}{c}3.52 \\
(17.00) \\
\end{array}$ & $\begin{array}{c}3.30 \\
(14.93) \\
\end{array}$ & $\begin{array}{c}3.03 \\
(12.40) \\
\end{array}$ \\
\hline $\begin{array}{l}\text { Drill sowing at } 20 \\
\mathrm{~cm}(\mathrm{ZT})\end{array}$ & $\begin{array}{c}4.87 \\
(26.47)\end{array}$ & $\begin{array}{c}3.10 \\
(13.27)\end{array}$ & $\begin{array}{c}2.90 \\
(11.33) \\
\end{array}$ & $\begin{array}{l}2.76 \\
(9.93) \\
\end{array}$ & $\begin{array}{l}2.49 \\
(7.80) \\
\end{array}$ & $\begin{array}{c}3.25 \\
(10.93) \\
\end{array}$ & $\begin{array}{c}3.74 \\
(19.13) \\
\end{array}$ & $\begin{array}{c}3.56 \\
(17.50) \\
\end{array}$ & $\begin{array}{c}3.35 \\
(15.27) \\
\end{array}$ & $\begin{array}{c}3.08 \\
(12.60) \\
\end{array}$ \\
\hline $\begin{array}{l}\text { Drill sowing at } 18 \\
\mathrm{~cm}(\mathrm{ZT})\end{array}$ & $\begin{array}{c}4.82 \\
(25.87) \\
\end{array}$ & $\begin{array}{c}3.05 \\
(12.80) \\
\end{array}$ & $\begin{array}{c}2.86 \\
(10.87) \\
\end{array}$ & $\begin{array}{l}2.69 \\
(9.40) \\
\end{array}$ & $\begin{array}{l}2.47 \\
(7.67) \\
\end{array}$ & $\begin{array}{c}3.34 \\
(11.67) \\
\end{array}$ & $\begin{array}{c}3.62 \\
(18.37) \\
\end{array}$ & $\begin{array}{c}3.49 \\
(16.80) \\
\end{array}$ & $\begin{array}{c}3.26 \\
(14.53) \\
\end{array}$ & $\begin{array}{c}2.99 \\
(11.93) \\
\end{array}$ \\
\hline $\begin{array}{l}\text { Bed planting ( } 3 \\
\text { rows) }\end{array}$ & $\begin{array}{c}4.84 \\
(26.13)\end{array}$ & $\begin{array}{c}3.17 \\
(14.13)\end{array}$ & $\begin{array}{c}2.95 \\
(11.47)\end{array}$ & $\begin{array}{c}2.79 \\
(10.01)\end{array}$ & $\begin{array}{c}2.53 \\
(8.07)\end{array}$ & $\begin{array}{c}3.28 \\
(11.13)\end{array}$ & $\begin{array}{c}3.75 \\
(19.20)\end{array}$ & $\begin{array}{c}3.60 \\
(17.60)\end{array}$ & $\begin{array}{c}3.37 \\
(15.60)\end{array}$ & $\begin{array}{c}3.12 \\
(13.20)\end{array}$ \\
\hline $\begin{array}{l}\text { Bed planting ( } 2 \\
\text { rows) }\end{array}$ & $\begin{array}{c}4.89 \\
(26.70)\end{array}$ & $\begin{array}{c}3.28 \\
(15.40)\end{array}$ & $\begin{array}{c}2.99 \\
(12.27)\end{array}$ & $\begin{array}{c}2.85 \\
(10.67)\end{array}$ & $\begin{array}{c}2.58 \\
(8.20)\end{array}$ & $\begin{array}{c}3.34 \\
(11.60)\end{array}$ & $\begin{array}{c}3.98 \\
(22.40)\end{array}$ & $\begin{array}{c}3.79 \\
(20.33)\end{array}$ & $\begin{array}{c}3.55 \\
(17.93)\end{array}$ & $\begin{array}{c}3.27 \\
(15.13)\end{array}$ \\
\hline SEm \pm & 0.02 & 0.037 & 0.03 & 0.03 & 0.02 & 0.04 & 0.04 & 0.03 & 0.02 & 0.03 \\
\hline $\mathrm{CD}$ at $5 \%$ & NS & 0.12 & 0.09 & 0.10 & 0.07 & NS & 0.13 & 0.10 & 0.07 & 0.09 \\
\hline \multicolumn{11}{|c|}{ Weed control treatments } \\
\hline PDN (50 g/ha) & $\begin{array}{c}5.81 \\
(32.78)\end{array}$ & $\begin{array}{c}5.78 \\
(32.67)\end{array}$ & $\begin{array}{c}5.33 \\
(27.56)\end{array}$ & $\begin{array}{c}4.97 \\
(23.89)\end{array}$ & $\begin{array}{c}4.40 \\
(18.56)\end{array}$ & $\begin{array}{c}3.85 \\
(13.89)\end{array}$ & $\begin{array}{c}6.71 \\
(44.39)\end{array}$ & $6.47(41)$ & $\begin{array}{c}6.11 \\
(36.44)\end{array}$ & $\begin{array}{c}5.59 \\
(30.33)\end{array}$ \\
\hline $\begin{array}{l}\text { CZN+MSM(RM) } \\
(25 \mathrm{~g} / \mathrm{ha})\end{array}$ & $\begin{array}{c}5.79 \\
(32.56) \\
\end{array}$ & $\begin{array}{c}1.59 \\
(1.56) \\
\end{array}$ & $\begin{array}{c}1.50 \\
(1.28)\end{array}$ & $\begin{array}{c}1.50 \\
(1.28) \\
\end{array}$ & $\begin{array}{c}1.32 \\
(0.78) \\
\end{array}$ & $\begin{array}{c}3.85 \\
(13.89) \\
\end{array}$ & $2.11(3.59)$ & $1.99(3.06)$ & $\begin{array}{c}1.79 \\
(2.28) \\
\end{array}$ & $1.62(1.67)$ \\
\hline $\begin{array}{l}\mathrm{PDN}+[\mathrm{CZN}+\mathrm{MSM} \\
(\mathrm{RM})] \\
(50+25 \mathrm{~g}) / \mathrm{ha}\end{array}$ & $\begin{array}{c}5.82 \\
(32.89)\end{array}$ & $\begin{array}{c}1.47 \\
(1.22)\end{array}$ & $\begin{array}{c}1.42 \\
(1.06)\end{array}$ & $\begin{array}{c}1.42 \\
(1.06)\end{array}$ & $\begin{array}{c}1.34 \\
(0.83)\end{array}$ & $\begin{array}{c}3.92 \\
(14.50)\end{array}$ & $2.13(3.64)$ & $1.93(2.78)$ & $\begin{array}{l}1.76 \\
(2.17)\end{array}$ & $1.61(1.61)$ \\
\hline Weed free & $\begin{array}{c}1.00 \\
(0.00)\end{array}$ & $\begin{array}{c}1.00 \\
(0.00)\end{array}$ & $\begin{array}{c}1.00 \\
(0.00)\end{array}$ & $\begin{array}{c}1.00 \\
(0.00)\end{array}$ & $\begin{array}{c}1.00 \\
(0.00)\end{array}$ & $1.00(0.00)$ & $1.00(0.00)$ & $1.00(0.00)$ & $\begin{array}{c}1.00 \\
(0.00)\end{array}$ & $1.00(0.00)$ \\
\hline Weedy check & $\begin{array}{c}5.82 \\
(32.92) \\
\end{array}$ & $\begin{array}{c}5.82 \\
(33.29) \\
\end{array}$ & $\begin{array}{c}5.31 \\
(27.28) \\
\end{array}$ & $\begin{array}{c}4.94 \\
(23.61) \\
\end{array}$ & $\begin{array}{c}4.50 \\
(19.39) \\
\end{array}$ & $\begin{array}{c}3.96 \\
(14.83) \\
\end{array}$ & $\begin{array}{c}6.79 \\
(45.56) \\
\end{array}$ & $\begin{array}{c}6.55 \\
(42.17) \\
\end{array}$ & $\begin{array}{c}6.16 \\
(37.22) \\
\end{array}$ & $\begin{array}{c}5.68 \\
(31.57) \\
\end{array}$ \\
\hline SEm \pm & 0.04 & 0.12 & 0.08 & 0.09 & 0.09 & 0.08 & 0.14 & 0.09 & 0.09 & 0.08 \\
\hline $\mathrm{CD}$ at $5 \%$ & 0.10 & 0.33 & 0.02 & 0.25 & 0.26 & 0.21 & 0.40 & 0.26 & 0.26 & 0.24 \\
\hline
\end{tabular}


Table.3 Effect of different herbicides and planting methods in wheat on weed dry matter accumulation, weed control efficiency and crop yield

\begin{tabular}{|c|c|c|c|c|c|c|c|c|c|c|c|c|c|}
\hline \multirow[t]{2}{*}{ Treatments } & \multicolumn{5}{|c|}{$\begin{array}{c}\text { Dry matter accumulation }\left(\mathrm{g} / \mathbf{m}^{2}\right) \\
\text { Grassy weeds }(\mathbf{G W})\end{array}$} & \multirow{2}{*}{\begin{tabular}{|c|}
$\begin{array}{c}\text { WCE (\%) } \\
\text { GW }\end{array}$ \\
At \\
harvest
\end{tabular}} & \multicolumn{5}{|c|}{$\begin{array}{l}\text { Dry matter accumulation }\left(\mathrm{g} / \mathrm{m}^{2}\right) \\
\text { Broad leaved weeds }(\mathrm{BLW})\end{array}$} & \multirow{2}{*}{\begin{tabular}{|c|}
$\begin{array}{c}\text { WCE (\%) } \\
\text { BLW }\end{array}$ \\
$\begin{array}{c}\text { At } \\
\text { harvest }\end{array}$ \\
\end{tabular}} & \multirow{2}{*}{$\begin{array}{l}\text { Wheat } \\
\text { grain } \\
\text { yield } \\
\text { (kg/ha) }\end{array}$} \\
\hline & $\begin{array}{c}\text { 30 } \\
\text { DAS }\end{array}$ & $\begin{array}{c}\text { 60 } \\
\text { DAS }\end{array}$ & $\begin{array}{c}90 \\
\text { DAS }\end{array}$ & $\begin{array}{c}\text { 120 } \\
\text { DAS }\end{array}$ & $\begin{array}{c}\text { At } \\
\text { harvest }\end{array}$ & & $\begin{array}{c}\text { 30 } \\
\text { DAS }\end{array}$ & \begin{tabular}{c|c} 
60 \\
DAS
\end{tabular} & \begin{tabular}{c|c}
90 \\
DAS
\end{tabular} & \begin{tabular}{l|l}
120 \\
DAS
\end{tabular} & $\begin{array}{c}\text { At } \\
\text { harvest }\end{array}$ & & \\
\hline \multicolumn{14}{|l|}{ Planting methods } \\
\hline Drill sowing at $20 \mathrm{~cm}(\mathrm{CT})$ & 3.41 & 9.77 & 24.44 & 43.95 & 55.11 & 59.4 & 11.92 & 57.36 & 103.90 & 127.69 & 121.96 & 59.3 & 5021.4 \\
\hline Drill sowing at $18 \mathrm{~cm}(\mathrm{CT})$ & 3.25 & 9.19 & 24.07 & 42.43 & 53.33 & 60.5 & 11.72 & 56.92 & 103.75 & 127.37 & 121.00 & 59.6 & 5143.1 \\
\hline Drill sowing at $20 \mathrm{~cm}(\mathrm{ZT})$ & 3.21 & 9.31 & 24.19 & 43.48 & 53.63 & 60.7 & 11.96 & 56.71 & 102.75 & 125.77 & 120.66 & 59.7 & 5042.2 \\
\hline Drill sowing at $18 \mathrm{~cm}(\mathrm{ZT})$ & 3.11 & 8.85 & 23.56 & 41.04 & 49.85 & 63.3 & 11.72 & 55.99 & 101.34 & 125.62 & 119.24 & 60.2 & 5161.4 \\
\hline Bed planting (3 rows) & 3.46 & 9.94 & 25.33 & 46.29 & 57.65 & 57.5 & 11.83 & 58.65 & 105.88 & 130.72 & 125.98 & 57.9 & 4876.3 \\
\hline Bed planting (2 rows) & 3.59 & 12.54 & 32.41 & 59.73 & 71.82 & 47.1 & 12.09 & 65.11 & 117.47 & 142.91 & 134.76 & 55.0 & 4438.0 \\
\hline SEm \pm & 0.18 & 0.74 & 2.13 & 3.01 & 3.71 & 4.9 & 0.10 & 0.51 & 0.90 & 1.26 & 0.65 & 1.1 & 134.2 \\
\hline $\mathrm{CD}$ at $5 \%$ & NS & 2.47 & 6.71 & 9.20 & 11.23 & 15.1 & NS & 1.60 & 2.84 & 3.97 & 2.04 & 3.5 & 412.3 \\
\hline \multicolumn{14}{|l|}{ Weed control treatments } \\
\hline PDN (50 g/ha) & 4.32 & 2.66 & 5.95 & 8.10 & 9.58 & 92.9 & 14.72 & 137.74 & 250.15 & 310.26 & 295.56 & 1.3 & 4797.1 \\
\hline $\mathrm{CZN}+\mathrm{MSM}(\mathrm{RM})(25 \mathrm{~g} / \mathrm{ha})$ & 4.38 & 21.33 & 58.10 & 107.61 & 131.45 & 3.2 & 14.83 & 6.34 & 12.71 & 14.00 & 12.44 & 95.8 & 5049.4 \\
\hline $\begin{array}{l}\mathrm{PDN}+[\mathrm{CZN}+\mathrm{MSM}(\mathrm{RM})] \\
(50+25 \mathrm{~g}) / \mathrm{ha}\end{array}$ & 4.45 & 2.28 & 4.89 & 6.32 & 6.53 & 93.2 & 14.97 & 6.22 & 11.75 & 13.81 & 12.30 & 95.9 & 5451.5 \\
\hline Weed free & 0.00 & 0.00 & 0.00 & 0.00 & 0.00 & 100.0 & 0.00 & 0.00 & 0.00 & 0.00 & 0.00 & 100.0 & 5585.3 \\
\hline Weedy check & 4.41 & 22.24 & 59.46 & 108.56 & 135.78 & 0.0 & 14.83 & 141.99 & 254.14 & 312.26 & 299.38 & 0.0 & 3846.0 \\
\hline SEm \pm & 0.27 & 2.03 & 4.44 & 5.58 & 6.39 & 7.1 & 0.25 & 4.37 & 5.83 & 6.48 & 9.09 & 1.6 & 86.1 \\
\hline $\mathrm{CD}$ at $5 \%$ & 0.86 & 5.76 & 13.32 & 17.11 & 19.88 & 21.1 & 0.72 & 12.42 & 16.57 & 18.42 & 25.86 & 4.5 & 271.3 \\
\hline
\end{tabular}


Similarly, WCE of both type of weeds increased with decreasing row spacing from $35 \mathrm{~cm}$ (bed planting with two rows) to $18 \mathrm{~cm}$ (drill sowing both in ZT and CT) (Table 3). Maximum WCE (both type of weeds) was recorded under zero tillage at $18 \mathrm{~cm}$ row spacing as compared to all other planting methods. Narrow spacing provided minimum space for weeds to grow; resulting thereby increased competitive potential of crop. Jat et al., (2003) recorded lower dry matter of both monocot and dicot weeds under close line (18 $\mathrm{cm})$ sowing as compared to normal line (22.5 $\mathrm{cm})$ sowing.

Wheat grain yield was significantly affected by various planting methods. The highest grain yield of wheat was observed under zero tillage at $18 \mathrm{~cm}$ row spacing (Table 3). Significantly lower grain yield of wheat was recorded in bed planting with two rows of wheat as compared to all other tillage treatments which remained at par with each other. The increase in grain yield under narrow row spacing could be attributed to lower weed infestation and more efficient utilization of available space (Table 3) as compared to wider row spacing in bed planting with two rows of wheat. The findings corroborated the results obtained by Jat et al., (2003) and Mali and Choudhary (2013).

\section{Effect of herbicides on weeds and crop}

Herbicidal treatments produced significantly lower weed density and their dry matter in comparison to weedy check treatment. Pinoxaden (50 g/ha) applied at $35 \mathrm{DAS}$, highly reduced the population and dry matter accumulation of grassy weeds like Phalaris minor at all growth stages after its application (Table 1, 2 and 3). Its effect was comparable to weed free and combined application of pinoxaden + [carfentrazone + metsulfuron $(\mathrm{RM})](50+25 \mathrm{~g}) /$ ha for the control of grassy weeds only with more than 90\% WCE. Sheoran et al., (2013) also reported that pinoxaden $(45 \mathrm{~g} / \mathrm{ha})$ provided excellent control $(95-100 \%)$ of grassy weeds.

Application of carfentrazone + metsulfuron (RM) $(25 \mathrm{~g} / \mathrm{ha})$ at $35 \mathrm{DAS}$, significantly reduced the population and dry matter accumulation of all broad leaved weeds having more than $95 \%$ WCE. Its effect was also comparable to weed free and combined application of pinoxaden + [carfentrazone + metsulfuron $(\mathrm{RM})](50+25 \mathrm{~g}) / \mathrm{ha}$ in respect of control of broad leaved weeds. Singh et al., (2011) also observed that premix of carfentrazone + metsulfuron $(25 \mathrm{~g} / \mathrm{ha}$ with $0.2 \%$ NIS) reduced the population of broad leaf weeds by 97-99 per cent.

Tank mixture of pinoxaden + [carfentrazone + metsulfuron $(\mathrm{RM})](50+25 \mathrm{~g}) / \mathrm{ha}$ at $35 \mathrm{DAS}$ was very effective against both grassy and broadleaf weeds with significantly lower weed density and their dry weight and more than $93 \%$ WCE, which might be due to synergistic effect of these herbicides in controlling both type of weeds. Similar findings have been reported by Singh et al., (2012) and Singh et al., (2010).

Herbicidal treatments, however, recorded significantly higher grain yield than weedy check (Table 3). The highest grain yield was observed under weed free treatment which was at par with the combined application of pinoxaden + [carfentrazone + metsulfuron (RM)] and was 45.21 and 41.73 per cent higher over weedy check, respectively. Alone application of pinoxaden and carfentrazone + metsulfuron (RM) increased the grain yield by 24.72 and 31.27 per cent, respectively, over weedy check, however, remained significantly lower as compared to the combined application of pinoxaden + [carfentrazone + metsulfuron (RM)] and weed free treatment (Table 3). Similar results have been reported by Sheoran et al., (2013). 


\section{References}

Balyan, R.S., Malik, R.K., Panwar, R.S. and Singh, S. 1991. Competitive ability of winter wheat cultivars with wild oat (Avena ludoviciana). Weed Sci., 39: 154-158.

Chhokar, R.S., Sharma, R.K. and Sharma, I. 2012. Weed management strategies in wheat: A review. J. Wheat Res., 4: 1-21.

Cochran, W.C. and Cox, G.M. 1959. Experimental designs, Asia Publishing House, Bombay.

Dev, D., Vivek, Singh, S.P. and Kumar, R. 2013. Weed management studies in wheat (Triticumaestivum) with herbicides under different establishment methods. Indian $J$. Agron., 58(2): 215-219.

Gomez, K.A. and Gomez, A.A. 1983. Statistical procedures for agricultural research, IRRI, A Wiley Pub., New York, pp: 199-201.

Jat, R.S., Nepalia, V. and Chaudhary, P.D. 2003. Influence of herbicides and methods of sowing on weed dynamics in wheat (Triticumaestivum L.). Indian J. Weed Sci., 35: 18-20.

Kapler, H., Lyon, D.J., Stahlman, P.W., Miller, S.D. and Eskridge, K.M. 2002. Wheat plant density influences jointed goatgrass (Aegilops cylindrica) competitiveness. Weed Tech., 16: 17-19.

Mali, M. and Choudhary, J. 2013. Performance of bread wheat (Triticum aestivum L.) varieties under different row spacing. J. Wheat Res., 4: 55-57.

Malik, R.K. and Singh, S. 1995. Littleseed canarygrass (Phalaris minor) resistance to isoproturon in India, Weed Tech., 9: 419-425.

Sharma, V. and Angiras, N.N. 1996. Effect of row orientation, row spacing and weed control methods on light interception, canopy temperature and productivity of wheat (Triticum aestivum L.), Indian J. Agron., 41: 390- 396.
Sheoran, S., Punia, S.S., Yadav, A. and Singh, S. 2013. Bioefficacy of pinoxaden in combination with other herbicides against complex weed flora in wheat. Indian J. Weed Sci., 45:90-92.

Singh, R., Singh, P., Singh, V.K., Singh, V.P. and Pratap, T. 2012. Effect of different herbicides on weed dry matter and yield of wheat, Int. Agron. Congr., IARI, New Delhi, Nov. 2630.2: 138-139.

Singh, S. 2007. Role of management practices on control of isoproturon-resistant littleseed canarygrass (Phalaris minor) in India. Weed Tech., 21: 339-346.

Singh, S., Malik, R.K., Balyan, R.S., and Singh, S. 1995. Distribution of weed flora of wheat in Haryana, Indian J. Weed Sci., 27: 114-121.

Singh, S., Punia, S. S, Yadav, A. and Hooda, V.S. 2011. Evaluation of carfentrazone-ethyl + metsulfuron-methyl against broadleaf weeds of wheat. Indian J. Weed Sci., 43: 12-22.

Singh, S., Punia, S. S. and Malik, R. K. 2009. Multiple resistance in isoproturon resistant biotypes of Phalaris minor in India. Weed Science Society of America 49th Annual Meeting and Southern Weed Science Society 62nd meeting, Hilton in Walt Disney World ${ }^{\circledR}$ Resort, Orlando, Florida, USA, Feb. 9-13, 2009. Abst. 280.

Singh, S., Yadav, A., Punia, S.S., Malik, R.S. and Balyan, R.S. 2010. Interaction of stage of application and herbicides on some Phalaris minor populations. Indian J. Weed Sci., 42: 144-154.

Walia, U.S., Brar, L.S. and Jand, S. 2003. Integrated effect of planting methods and herbicides on Phalaris minor and wheat. Indian J. Weed Sci., 35: 169-172.

Wrubel, R.P. and Gressel, J. 1994. Are herbicide mixtures useful for delaying the rapid evolution of resistance? a case study. Weed Tech., 8: 635-648.

\section{How to cite this article:}

Navish Kumar Kamboj, V.S. Hooda, Gaurendra Gupta, Meenakshi Sangwan. 2017. Weed Management Studies in Wheat (Triticum aestivum) with Herbicides under Different Planting Methods. Int.J.Curr.Microbiol.App.Sci. 6(2): 1742-1749.

doi: http://dx.doi.org/10.20546/ijcmas.2017.602.195 